\title{
Feature-level Fusion of Dual-band Infrared Images Based on Gradient Pyramid Decomposition
}

\author{
Xiujie Qu,Fu Zhang \\ School of Information and Electronics \\ Beijing Institute of Technology \\ Beijing, China \\ e-mail: zhangfu_raymond@yahoo.cn
}

\author{
Ying Zhang \\ School of Information and Electronics \\ Beijing Institute of Technology \\ Beijing, China
}

\begin{abstract}
Infrared thermal imager has been widely used in the fields of missile guidance and flaw detection. To identify the target clearly, the advanced one adopts dual bands sensors to capture images. Since of that, there is an urgent need of a fusion of the dual-bands images. The fused result includes much more exhaustive information than any single one, and can better reflect the actual. Among the algorithms used to fuse the dual-band infrared images, the weighted algorithm is the most widely used and easiest to be achieved. Nonetheless, its effect is not desired. We extract the features of the source images and make a fuse based on them on the feature-level. To get a better result, in this paper, the fusion strategy based on the Gradient pyramid transform has been mainly adopted. Meanwhile, there is a comparison with the weighted algorithm. Also, it makes an evaluation and analysis to the experimental data, and finally obtains the desired results.
\end{abstract}

Keywordst-image fusion, feature extraction, Gradient pyramid decomposition, image reconstruction

\section{INTRODUCTION}

Infrared thermal imager has been widely used in the domains of missile guidance and flaw detection. Since putting the imager into operation, the single-band infrared detectors show some limitations in detecting targets. On the one hand, the target sometimes cannot be discovered by medium wave infrared (MWIR) detector but can be easily detected by long wave infrared (LWIR) one. On the other hand, the detection-range of MWIR is much longer then LWIR in a humid environment [6]. To improve the detection results, the advanced imager adopts sensors of dual bands to identify the target and extract the feature of it. Feature detection is a low-level image processing operation. That is, it is usually performed as the first operation on an image, and examines every pixel to see if there is a feature present at that pixel. If this is part of a larger algorithm, then the algorithm will typically only examine the image in the region of the features [7]. The fused result includes much exhaustive information than any single one that takes part in the fusion and can better reflect the actual $[1,4]$. Therefore, it can greatly enhance the definition of image and effectively improve the success rate of detection of different classes of target.

In the choice of dual-band infrared images fusion algorithm, the weighted algorithm is the most simple and commonly used one based on spatial domain. It is characterized by simple and intuitive, suitable for real-time processing. However, at the same time, its essence is a smoothing of the image processing. Therefore, the edges and the contours of the image will become blurred. Making matters worse, when the difference of the images is great, there will be traces of mosaic, which is not conductive to the human eye to identify [5].

Compared with the weighted algorithm, the Gradient pyramid decomposition is a kind of fusion algorithms based on the transformation domain. It uses 4 Gradient operators, based on the Gaussian pyramid decomposition, to make a filtering in the horizontal, vertical, and two diagonal directions. By this way, we can better extract the edge information of the source image, and keep the details of the characteristics. The fused image has a better definition and contains enough effective messages. In this paper, we do a comparative experiment of dual-bands infrared image fusion. One group takes the Gradient pyramid algorithm, and the other is based on the weighted algorithm. Through making an analysis and evaluation of the experimental data, we have achieved the desired results.

\section{WEIGHTED FUSION ALGORITHM}

Weighted average method is a weighted average processing of two registration source images on the corresponding pixels [4]. Set $A(x, y)$ and $B(x, y)$ are the two pixels of source images, the fusion result is $F(x, y)$, the process of weighted fusion can be expressed as:

$$
\begin{gathered}
F(x, y)=\omega_{1} A(x, y)+\omega_{2} B(x, y) \\
\omega_{1}+\omega_{2}=1
\end{gathered}
$$

$x, y$ represent the row and column numbers of the image, $\omega_{1} 、 \omega_{2}$ denote the weighted coefficient. In applications, the weights can be obtained by principal component analysis: first step, calculate the covariance of two images matrix:

$$
C=\left[\begin{array}{ll}
v_{A} & c_{A B} \\
c_{A B} & v_{B}
\end{array}\right]
$$


and then calculate the eigenvalues of the covariance matrix $\lambda_{1}, \lambda_{2}$.After that, we can find out the largest eigenvalue, , then the eigenvectors $X=\left(\begin{array}{ll}x_{1} & x_{2}\end{array}\right)^{-1}$,according to the formula:

$$
\left(\begin{array}{lr}
\lambda_{\max }-v_{A} & -c_{A B} \\
-C_{A B} & \lambda_{\max }-v_{B}
\end{array}\right) X=0
$$

Finally, we can get the nonzero solution. When using PCA method to determine the weighting coefficient, the part of great gray gradient of the image will be enhanced. Therefore, this method is of significant effect in processing the images of huge difference in the gray gradient. However, this method is subject to the noise impact, especially the impulse noise. The gray scale mutations can even make the fusion results worse.

\section{GRADIENT PYRAMID DECOMPOSITION}

The Gradient pyramid decomposition is a kind of multiresolution algorithm based on the Gaussian decomposition. The process steps are as follow:

\section{A. Establish Gaussian pyramid}

In the image sequence of Gauss pyramid, through a low pass filter to the former one and then making a down sampling, each level is obtained.

$$
G_{l}(i, j)=\sum_{m=-2}^{2} \sum_{n=-2}^{2} \omega(m, n) G_{l-1}(2 i+m, 2 j+m)
$$

In the formula, $G_{l}(i, j)$ denotes the $L$-level image of Gaussian pyramid, $G_{0}$ denotes the source image as the bottom of the pyramid. $N$ is the level number and $C_{l}$ means the column number while $R_{l}$ means the row number of the Lth level. $\omega(m, n)=h(m) \bullet h(n)$ is a window function of lowpass characteristics with $5 * 5$ in size. $h$ means Gauss density distribution function, we can calculate the value of $h$ :

$$
h(0)=\frac{3}{8}, h(-1)=h(+1)=\frac{1}{4}, h(-2)=h(+2)=\frac{1}{16}
$$

Finally, we get the sequences consists of $G_{0}, G_{1}, \ldots, G_{N}[2,3]$.

\section{B. Establish Gradient pyramid}

We can get the Gradient pyramid sequence by making a four-gradient-direction filter of each level of the Gaussian pyramid:

$$
G P_{k l}=d_{l}^{*}\left[G_{k}+\dot{\omega} * G_{k}\right]
$$

Here $G P_{k l}$ is the $K$-level and $L$-direction Gradient pyramid ( $l=1,2,3,4$ respectively mean the horizontal, vertical, two diagonal directions), $G_{k}$ is the Kth level image of Gaussian pyramid, $d_{l}$ is the $L$ th direction gradient operator. $\dot{\omega}$ is a core in size of $3^{*} 3$. The filter operator can be defined as follows:

$$
\begin{aligned}
& d_{1}=\left[\begin{array}{ll}
1 & -1
\end{array}\right], \quad d_{2}=\frac{1}{\sqrt{2}}\left[\begin{array}{rr}
0 & -1 \\
1 & 0
\end{array}\right] \\
& d_{3}=\left[\begin{array}{r}
-1 \\
1
\end{array}\right], \quad d_{4}=\frac{1}{\sqrt{2}}\left[\begin{array}{rr}
-1 & 0 \\
0 & 1
\end{array}\right]
\end{aligned}
$$

The $\omega$ is defined as:

$$
\dot{\omega}=\frac{1}{16}\left[\begin{array}{ccc}
1 & 2 & 1 \\
2 & 4 & 2 \\
1 & 2 & 1
\end{array}\right]
$$

Each level of the Gradient contains details on the horizontal, vertical, and diagonal directions. Therefore, we can extract more clear and exhaustive message from the source image, and preserve the edge feature.

\section{Reconstruction of the Gradient pyramid}

The reconstruction of the Gradient pyramid is somewhat complicated. The directed Laplacian pyramid and filter subtract decimate (FSD) Laplacian pyramid are generated as the intermediate results during the process. The directed Laplacian pyramid is defined as:

$$
\overrightarrow{L_{k l}}=-\frac{1}{8} d_{l} * G P_{k l}
$$

$\overrightarrow{L_{k l}} G P_{k l}$ is the $K$-level and $L$-direction of the directed Laplacian pyramid. Its accumulation forms FSD Laplacian pyramid $L_{k}$ :

$$
L_{k}=\sum_{l=1}^{4} \overrightarrow{L_{k l}}
$$

And the Laplacian is recovered from FSD pyramid:

$$
L P_{k}=[1-\omega]^{*} L_{k}
$$

Finally we can get the fused image by reconstructing the Laplacian pyramid [2].

D. Steps of the image fusion algorithm

- Do Gaussian decomposition of each source image, and establish the pyramid sequences. 
- $\quad$ Establish the Gradient pyramid sequences based on the Gaussian pyramid.

- Fuse the pyramid decomposition layer with appropriate rule to obtain the fused image pyramid.

- Reconstruct the fused image pyramid, and then obtain the final fused image.

\section{FEATURE EXTRACTION}

In this paper, we take the sobel operator to extract the features of the MWIR source image and the canny one to get the features of the LWIR image.

\section{A. Canny operator}

The Canny edge detector is an edge detection operator that uses a multi-stage algorithm to detect a wide range of edges in images. The extraction results can have a clear contour and prolific details of the target. The extraction steps of the canny operator are as follow:

a) Noise reduction: The image after a Gaussian mask has been passed across each pixel. Because the Canny edge detector is susceptible to noise present in raw unprocessed image data, it uses a filter based on a Gaussian, where the raw image is convolved with a Gaussian filter.

b) Finding the intensity gradient of the image: An edge in an image may point in a variety of directions, so the Canny algorithm uses four filters to detect horizontal, vertical and diagonal edges in the blurred image. The edge detection operator returns a value for the first derivative in the horizontal direction $\left(G_{x}\right)$ and the vertical direction $\left(G_{y}\right)$. From this the edge gradient and direction can be determined:

$$
\begin{gathered}
M[\mathrm{x}, \mathrm{y}]=\sqrt{G_{x}(x+y)^{2}+G_{y}(x+y)^{2}} \\
\theta[\mathrm{x}, \mathrm{y}]=\arctan \sqrt{G_{x}(x+y) / G_{y}(x+y)}
\end{gathered}
$$

c) Non-maximum suppression: The same binary map shown on the left after non-maxima suppression. Given estimates of the image gradients, a search is then carried out to determine if the gradient magnitude assumes a local maximum in the gradient direction.

d) Tracing edges through the image and hysteresis thresholding: Large intensity gradients are more likely to correspond to edges than small intensity gradients. It is in most cases impossible to specify a threshold at which a given intensity gradient switches from corresponding to an edge into not doing so. Therefore Canny uses thresholding with hysteresis.

\section{B. Sobel operator}

The Sobel operator is used in image processing, particularly within edge detection algorithms. Technically, it is a discrete differentiation operator, computing an approximation of the gradient of the image intensity function.
Mathematically, the operator uses two $3 \times 3$ kernels which are convolved with the original image to calculate approximations of the derivatives - one for horizontal changes, and one for vertical. If we define $\mathrm{A}$ as the source image, and $G_{x}$ and $G_{y}$ are two images which at each point contain the horizontal and vertical derivative approximations, the computations are as follows:

$$
G_{x}=\left[\begin{array}{ccc}
-1 & 0 & 1 \\
2 & 0 & 2 \\
1 & 0 & 1
\end{array}\right] * A \quad G_{y}=\left[\begin{array}{ccc}
1 & 2 & 1 \\
0 & 0 & 0 \\
-1 & -2 & -1
\end{array}\right] * A
$$

\section{EVALUATION CRITERIA}

\section{A. Information entropy}

The concept of information entropy was proposed by Shannon, the famous scientist who was the founder of Information Theory. It indicates the amount of information in the signal.The information entropy can be described as:

$$
E=-\sum_{i=0}^{L} P(l) \log _{2} P(l)
$$

$P(l)$ is the probability of a gray value $l$ appears in the image, $L$ is the image gray scale. The value of entropy in the fused image is the bigger the better. It means the fused result contains more effective information.

\section{B. Average gradient}

Average gradient can be expressed to the improvement of image quality. It reflects the clarity of the image, and also reflects the contrast of small details in the image and the features of veins.

\section{Standard deviation}

The standard deviation reflects the degree of dispersion of the gray scale relative to the average value. The standard deviation is the greater, the image gray level distribution is more dispersed, and there is more identifiable information.

\section{Edge intensity}

The edge intensity is a kind of evaluation criteria, which is used to evaluate the preservation of the edge features of the source image in the fused results. The value of it is the bigger, there is more edge information in the fused image [7].

\section{ANALYSIS OF EXPERIMENTAL RESULTS}

In this paper, we use two wavebands infrared registration images with relatively clean background by an enhancement and de-noising process. The Gradient pyramid and weighted and the Contrast have been adopted to do a comparative experiment of the fusion result on the feature-level. We give an objective evaluation of the fused image used the above criteria, and finally achieve the desired experimental results.

Judging from the visual effects, the figure of Gradient pyramid decomposition can reflect more clearly the outline characteristics of pedestrians and the warship, compared with 
the weighted method. Also it can reduce the interference of target identification caused by the thermal radiation of the beach, avoid the ghosting caused by the weighted algorithm.

Judging from the evaluation criteria, the value of average gradient and edge intensity of the Gradient pyramid is higher then the weighted. Therefore, the fused image using Gradient pyramid decomposition can reflect more details of the target, and this is conducive to identify the target of small feature size.

TABLE I. THIS IS THE CRITERIA OF THE FUSION.

\begin{tabular}{|c|c|c|}
\hline & The Gradient & The weighted \\
\hline Information entropy & 1.7822 & 1.3717 \\
\hline Average gradient & 9.0018 & 6.6617 \\
\hline Standard deviation & 10.2647 & 7.5182 \\
\hline Edge intensity & 79.0321 & 58.2687 \\
\hline $\begin{array}{c}\text { Runtime } \\
\text { (MATLAB 2007B) }\end{array}$ & 0.0958 & 0.0821 \\
\hline
\end{tabular}

REFERENCES
[1] Guoqiang Ni. Study on Multi-band Image Fusion Algorithms and Its Progressing(I).[J]. Optoelectronic Technology \& Information, 2001,14 (5) :11 -17.J. Clerk Maxwell, A Treatise on Electricity and Magnetism, 3rd ed., vol. 2. Oxford: Clarendon, 1892, pp.68-73.

[2] Clark, G.A.,Sengupta, S.K.,Institute of Electric and Electronic Engineer et al.Detecting buried objects by fusing dual-band infrared images[C].//Signals, Systems and Computers, 1993. 1993 Conference Record of The Twenty-Seventh Asilomar Conference on vol.1.1993:135-143.

[3] Jianlin Li, Jiancheng Yu. Study of Image Fusion Based on Grad Pyramid Algorithm [J]. Science Technology and Engineering, 2007,7 (22) :5818-5822.

[4] Mingge Xia, You He, Xiaoming Tang. The fusion of the development status and Prospects [J]. Ship Electronic Engineering, 2002, (6) :2 -13, 29.

[5] Ting Wang. Study on Infrared and glimmer Image Fusion [D]. Nanjing University of Technology and Engineering, 2007.

[6] Alexander M. Akhmetshin,Lyudmila,G. Akhmetshin et al.Sensitive segmentation of low contrast multispectral image on base multiparameter space-resonance imaging method[C].//Intelligent Robots and Computer Vision XX: Algorithms, Techniques, and Active Vision.2001:279-289.

[7] Zhongliang Jing, Gang Xiao, Zhenhua Li. Image Fusion - Theory and Applications. Higher Education Press, 2007:

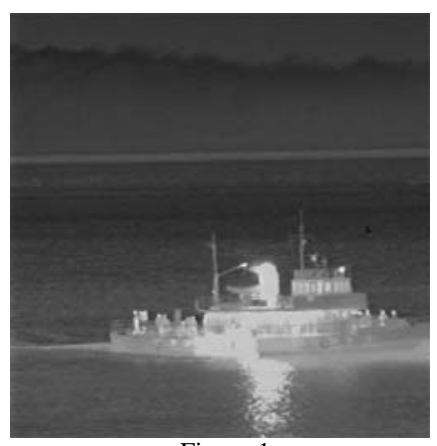

Figure 1

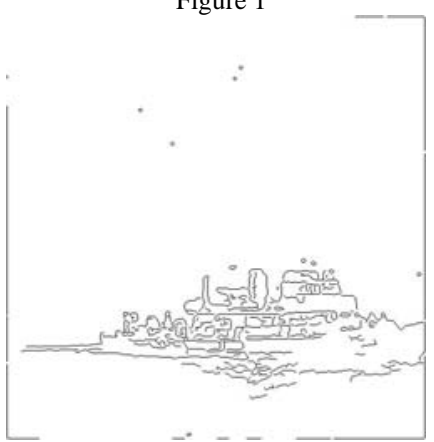

Figure 4

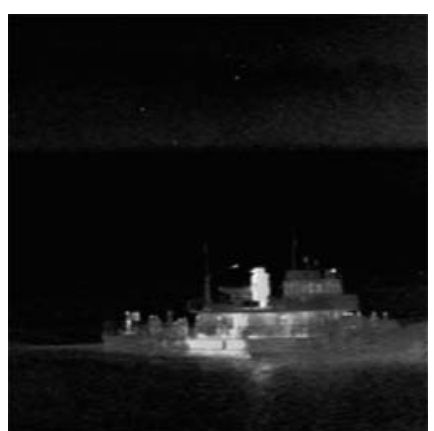

Figure 2

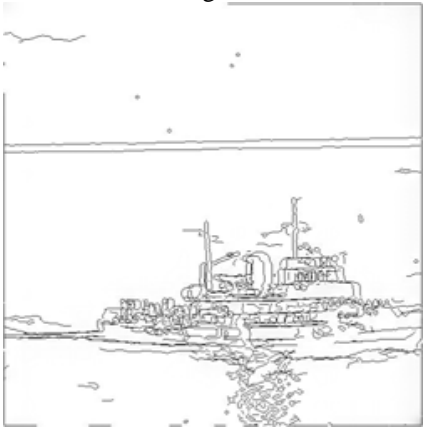

Figure 5

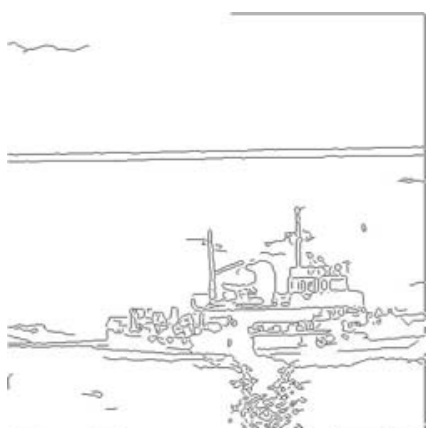

Figure 3

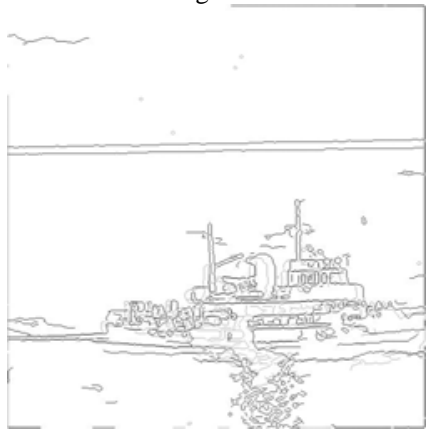

Figure 6

Figure: (1) is the LWIR source image; (2) is the MWIR image; (3) is the edge extracted of the LWIR; (4) is the edge extracted of the NWIR; (5) is the fusion image in the Gradient Pyramid algorithm;(6) is the fusion image in the weighted algorithm. 